\title{
A doença de Alzheimer, hoje
}

Alexandre de Mendonça*

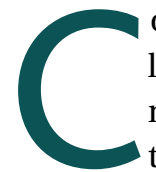
om o envelhecimento da população, a prevalência da doença de Alzheimer e de outras formas de declínio cognitivo tem vindo a aumentar. Estima-se que haja 5 milhões de pessoas com demência na Europa. ${ }^{1}$ Estas situação torna-se, pois, cada vez mais frequente na prática diária do clínico geral. Pareceu-me assim relevante, e tive muito gosto em aceitar, o honroso convite do Editor da Rev Port Clin Geral, o meu colega de curso e amigo Mestre António Faria Vaz, para elaborar este número especial da revista, com a colaboração inestimável de vários especialistas, nomeadamente do Grupo de Demências da Faculdade de Medicina de Lisboa e Instituto de Medicina Molecular. O objectivo geral será propor ao clínico geral orientações práticas em relação aos problemas que têm de resolver nos seus doentes com declínio cognitivo. A este respeito o artigo dos Drs. Rui Pereira Alves e Ana Isabel Caetano sobre O papel do clínico geral no seguimento dos doentes com declínio cognitivo é com certeza muito útil. Mas acredito ser não menos importante expor ao clínico geral o cenário de rápida transformação a que se assiste na área da doença de Alzheimer, com consequências em termos diagnósticos e terapêuticos que se estão progressivamente a reflectir na prática clínica.

A doença de Alzheimer é uma doença degenerativa de início insidioso e evolução progressiva, cuja prevalência aumenta com a idade. É caracterizada por degenerescência e morte neuronal, em relação com a deposição no cérebro de várias substâncias, notadamente o péptido beta-amilóide e a proteína tau, e atinge inicialmente áreas cerebrais, como o hipocampo e estruturas relacionadas na parte interna do lobo temporal, importantes para os processos de memória. ${ }^{2} \mathrm{~A}$ doença de Alzheimer é a causa mais frequente de demência. De facto, para se diagnosticar doença de Alzheimer presentemente, pelos critérios de diagnóstico reconhecidos, nomeadamente da International Classification of $\mathrm{Di}$ seases, ${ }^{3}$ Diagnostic and Statistical Manual of Mental Di-

*Grupo de Demências, Serviço de Neurologia e Laboratório de Neurociências, Faculdade de Medicina e Instituto de Medicina Molecular, Lisboa. sorders $^{4}$ ou National Institute of Neurological and Communicative Disorders and Stroke, ${ }^{5}$ é requerida a existência de demência, ou seja, o doente terá de apresentar défices da memória e de outras funções cognitivas, que representem declínio em relação a um nível prévio, e interfiram significativamente com a sua actividade social e profissional. Porém, a doença de Alzheimer inicia-se, de forma insidiosa, por via de regra com esquecimentos, muitos anos antes de o doente chegar a um grau de declínio cognitivo e funcional compatível com o diagnóstico de demência. De resto, é muitas vezes difícil valorizar as queixas iniciais de memória na fase inicial da doença de Alzheimer: por um lado, mesmo as pessoas saudáveis têm frequentemente uma impressão pouco favorável em relação à sua memória; por outro lado, o próprio processo de envelhecimento se pode acompanhar de um ligeiro declínio objectivo da memória. ${ }^{6}$ Neste contexto, várias entidades nosológicas, com algumas diferenças conceptuais, foram propostas, nas últimas décadas, para descrever os doentes que apresentam declínio cognitivo sem demência. ${ }^{7}$ Destas entidades, a que se tornou indubitavelmente mais popular foi o Defeito Cognitivo Ligeiro (Mild Cognitive Impairment), definida por Petersen e colaboradores, ${ }^{8} \mathrm{e}$ aperfeiçoada ulteriormente. ${ }^{9}$

Parece-me indubitável que, sobretudo para os neurologistas interessados em problemas cognitivos, o enquadramento dos doentes, cada mais novos e com queixas cognitivas mais subtis, tomando como referência o conceito nosológico de Defeito Cognitivo Ligeiro, tem sido muito útil, permitindo estabelecer, nesses casos, uma probabilidade elevada de progressão para demência, e propor um seguimento cuidado. No entanto, o conceito nosológico de Defeito Cognitivo Ligeiro apresenta limitações. Em primeiro lugar, parece corresponder mais a um estádio evolutivo do que a uma doença. ${ }^{10}$ Em segundo lugar, alguns doentes com Defeito Cognitivo Ligeiro mantêm-se intrigantemente estáveis durante muitos anos sem evoluirem para demência. ${ }^{11}$ Em terceiro lugar, como indicámos num artigo recente, alguns pacientes com queixas cognitivas sem alterações demonstráveis na avaliação neuropsicológica, 
e que não preenchem por isso os critérios de Defeito Cognitivo Ligeiro, evoluem, mesmo assim, para demência. ${ }^{12}$ Assim, o conceito de Defeito Cognitivo Ligeiro é insatisfatório em termos de diagnóstico e prognóstico.

Porém, e talvez mais importante, os estudos realizados na última década com os pacientes diagnosticados de Defeito Cognitivo Ligeiro permitiram compreender melhor o desenvolvimento inicial da doença de Alzheimer, e criar as condições para propor novos critérios de doença de Alzheimer que estabelecem o diagnóstico em fases precoces, sem necessidade de o doente estar demente. ${ }^{13}$ Estes critérios são ainda considerados apropriados para o contexto de investigação, mas a verdade é que a sua aplicação permeia já vários aspectos da assistência clínica especializada. Essencialmente, os novos critérios baseiam-se na identificação de alterações patológicas da doença de Alzheimer nas regiões afectadas, ou biomarcadores, nomeadamente: 1) declínio gradual e progressivo da memória, objectivado por testes neuropsicológicos de memória episódica 2) atrofia do hipocampo e estruturas cerebrais relacionadas, detectada por técnicas volumétricas de ressonância nuclear magnética 3) alterações específicas do péptido beta-amilóide, proteína tau total e proteína tau hiperfosforilada no liquor, obtido por punção lombar 4) hipometabolismo temporal e parietal bilateral, revelado com tomografia de emissão de positrões (PET). Em casos familiares, a detecção de mutações nos genes que causam doença autossómica dominante pode contribuir para estabelecer o diagnóstico definido de doença de Alzheimer. ${ }^{13}$ De salientar, ainda, que outros biomarcadores, genéticos, bioquímicos e imagiológicos com recurso a radioligandos com afinidade para o beta-amilóide, se poderão revelar importantes no futuro próximo.

Neste momento, falta saber qual é a combinação de biomarcadores mais sensível e específica para o diagnóstico da doença de Alzheimer em fase precoce. Estudos multicêntricos estão em curso para responder a esta importante questão, em particular no âmbito do programa Alzheimer's Disease Neuroimaging Initiative (ADNI). ${ }^{14}$ No Grupo de Demências da Faculdade de Medicina de Lisboa e Instituto de Medicina Molecular, um dos aspectos que nos tem interessado é melhorar a contribuição diagnóstica dos testes neuropsicológicos, quer empregando provas de memória muito exigentes, de que é exemplo o Teste de Aprendizagem Verbal de Califórnia (California Verbal Learning Test, CVLT), ${ }^{15,16}$ quer recorrendo a modelos estatísticos avançados englobando provas seleccionadas em vários domínios cognitivos. Por outro lado, o estabelecimento de testes rápidos de rastreio de declínio cogntivo será essencial para o clínico geral fazer a identificação precoce destes doentes. Este aspecto é abordado no artigo da Prof. Manuela Guerreiro, intitulado Testes de Rastreio de Defeito Cognitivo e Demência: Uma perspectiva prática. Outra vertente promissora consiste em investigar as pequenas alterações que os doentes apresentam nas suas capacidades funcionais do dia-a-dia. A utilização de escalas de actividades de vida diária, especialmente concebidas para estas fases iniciais da doença, poderá identificar, com uma sensibilidade e especificidade elevadas, alterações funcionais subtis nas actividades de vida diária dos pacientes que sofrem de doença de Alzheimer incipiente. ${ }^{17}$

Claramente, o processo diagnóstico da doença de Alzheimer está numa fase de dramática transformação. É possível hoje em dia detectar precocemente as alterações patológicas típicas da doença de Alzheimer, porém as técnicas envolvidas são pouco disponíveis, complexas, dispendiosas, e nalguns casos invasivas. A sua utilização terá certamente de ser seleccionada com rigor, no sentido de serem facultadas a pacientes que delas possam realmente beneficiar. Doentes mais jovens, ou que precisem tomar decisões de carácter profissional ou familiar, serão exemplos. Também o diagnóstico preciso e precoce será importante em pacientes candidatos a participarem em ensaios clínicos.

No que diz respeito à terapêutica da doença de Alzheimer, é verdade que não têm sido introduzidos recentemente novos medicamentos específicos, para além dos inibidores da acetilcolinesterase e do antagonista dos receptores do glutamato de subtipo $N$-metilD-aspartato (NMDA), memantina. Mas tem havido progressos no que diz respeito à terapêutica mais judiciosa dos Sintomas psiquiátricos e comportamentais da demência, revista pelo Dr. Frederico Simões do Couto e por mim próprio nesta revista, e bem assim ao importante aspecto relacionado com a saúde e A Educação dos Cuidadores, abordado pela Dra. Sofia Madureira. Mas a grande esperança terapêutica na doença de Alzheimer reside nos diferentes fármacos que interfe- 
rem com os processos metabólicos associados ao péptido beta-amilóide, de que é exemplo o anticorpo monoclonal bapineuzumab, ${ }^{18} \mathrm{e}$ que se espera possam atenuar ou idealmente interromper a progressão da patologia. Vários ensaios clínicos decorrem, ou estão planeados actualmente, no nosso país, com estes fármacos. Face a uma situação tão destrutiva e inexorável como a doença de Alzheimer, a possibilidade de efectuar um diagnóstico precoce e de tentar uma terapêutica modificadora da doença constitui, hoje em dia, para os doentes, as famílias e os profissionais de saúde envolvidos, uma esperança notável.

Idealmente, o objectivo último será prevenir ou adiar significativamente a doença de Alzheimer. Se já se conhece muito acerca de factores de risco ou protecção associados à doença de Alzheimer, a verdade é que esse conhecimento assenta em grande parte em estudos epidemiológicos, ou em ensaios clínicos concebidos com outros objectivos. Só recentemente se começaram grandes estudos de prevenção primária com o objectivo específico de avaliar o papel de várias intervenções sobre a emergência de declínio cognitivo ou demência. Este tema de grande interesse é revisto pela Dra. Ana Verdelho no artigo Prevenção do defeito cognitivo e demência - os factores de risco cardio-cerebro-vasculares. Uma noção parece certa, a preparação do envelhecimento cerebral terá de começar muito cedo na vida.

\section{REFERÊNCIAS BIBLIOGRÁFICAS}

1. Olesen J, Baker MG, Freund T, di Luca M, Mendlewicz J, Ragan I, Westphal M (2006) Consensus document on European brain research. J Neurol Neurosurg Psychiatry 77 Suppl 1:11-49.

2. Blennow K, de Leon MJ, Zetterberg H (2006) Alzheimer's disease. Lancet 368:387-403.

3. World Health Organization. The ICD-10 Classification of Mental and Behavioural Disorders, World Health Organization, 1992, Geneva, Switzerland.

4. American Psychiatric Association. Diagnostic and Statistical Manual of Mental Disorders, $4^{\text {a }}$ ed., American Psychiatric Association, 1994, Washington.

5. McKhann G, Drachman D, Folstein M, Katzman R, Price D, Stadlan, EM. Clinical diagnosis of Alzheimer's disease: Report of the NINCDS ADRDA Work Group. Neurology 198434 939-944.

6. Mendes T, Ginó S, Ribeiro F, Guerreiro M, de Sousa G, Ritchie K, de Mendonça A. Memory complaints in healthy young and elderly adults: reliability of memory reporting Aging Ment Health 2008 12:177-182.

7. Ribeiro F, Guerreiro M, de Mendonça A. Defeito cognitivo ligeiro, in Psicogeriatria, Firmino H, Cortez Pinto L, Leuschner A, Barreto J (eds.). Edi- tora Psiquiatria Clínica, pp 315-331, 2006, Coimbra.

8. Petersen RC, Smith GE, Waring SC, Ivnik RJ, Tangalos EG, Kokmen E (1999) Mild cognitive impairment. Arch Neurol 1999 56:303-308.

9. Portet F, Ousset PJ, Visser PJ, et al. MCI Working Group of the European Consortium on Alzheimer's Disease (EADC). Mild cognitive impairment $(\mathrm{MCl})$ in medical practice: a critical review of the concept and new diagnostic procedures. Report of the $\mathrm{MCl}$ Working Group of the European Consortium on Alzheimer's disease. J Neurol Neurosurg Psychiatry 2006 77:714-718.

10. Gauthier S, Reisberg B, Zaudig M, Petersen RC, Ritchie K, Broich K, Belleville $S$, Brodaty $H$, Bennett $D$, Chertkow $H$, Cummings JL, de Leon $M$, Feldman H, Ganguli M, Hampel H, Scheltens P, Tierney MC, Whitehouse $P$, Winblad B; International Psychogeriatric Association Expert Conference on mild cognitive impairment. Mild cognitive impairment. Lancet 2006 367:1262-1270.

11. Petersen RC, Doody R, Kurz A, Mohs RC, Morris JC, Rabins PV, Ritchie $K$, Rossor M, Thal L, Winblad B. Current concepts in mild cognitive impairment. Archives of Neurology 2001 58:1985-1992.

12. Nunes T, Fragata I, Ribeiro F, Palma T, Maroco J, Cannas J, Secca M, Menezes C, Carmo I, Cunha G, Castelo Branco M, Guerreiro M, de Mendonça $A$. The outcome of elderly patients with cognitive complaints but normal neuropsychological tests. J Alzheim Dis 2010 19:137-145.

13. Dubois B, Feldman HH, Jacova C, Dekosky ST, Barberger-Gateau P, Cummings J, Delacourte A, Galasko D, Gauthier S, Jicha G, Meguro K, O'brien J, Pasquier F, Robert P, Rossor M, Salloway S, Stern Y, Visser PJ, Scheltens P.Alzheimer's Disease Neuroimaging Initiative

14. Alzheimer's Disease Neuroimaging Initiative, http://www.adni-info.org/.

15. Delis DC, Kramer JH, Kaplan E, Ober BA. California Verbal Learning Test: Research edition, The Psychological Corporation, 1987, New York.

16. Baeta E. Bateria para a avaliação neuropsicológica de adultos com epilepsia. Psicologia 2002 16:79-96.

17. Pedrosa H, de Sá A, Guerreiro M, Maroco J, Simões MR, Galasko D, de Mendonça A. Functional Evaluation distinguishes $\mathrm{MCl}$ patients from healthy elderly people - the ADCS/MCI/ADL scale. J Nutr Health Aging 2010 (in press).

18. Salloway S, Sperling R, Gilman S, Fox NC, Blennow K, Raskind M, Sabbagh M, Honig LS, Doody R, van Dyck CH, Mulnard R, Barakos J, Gregg KM, Liu E, Lieberburg I, Schenk D, Black R, Grundman M; For the Bapineuzumab 201 Clinical Trial Investigators. A phase 2 multiple ascending dose trial of bapineuzumab in mild to moderate Alzheimer disease. Neurology 2009 73:2061-2070.

O autor declarou não possuir conflitos de interesse.

\section{ENDEREÇO PARA CORRESPONDÊNCIA}

Alexandre de Mendonça

Laboratório de Neurociências

Instituto de Medicina Molecular

Av. Prof. Egas Moniz

1649-028 Lisboa

E-mail: mendonca@fm.ul.pt. 